\title{
Analysis of the event of 2004 November 10
}

\author{
Yuan Ma and LiYing Zhu \\ Yunnan Astronomical Observatory/National Astronomical Observatories, Chinese Academy of \\ Sciences, Kunming, 650011 \\ email: mayuan@ynao.ac.cn
}

\begin{abstract}
The large burst of complex type which occurred at the metric, decimetric and microwave wavelengths from 0203 to 0304 UT on 2004 November 10 is analyzed in this present article, some prominent features of this event are obtained from the correlative analysis of this event with the $\mathrm{H} \alpha$ flare and coronal mass ejection ( $\mathrm{CME}$ ) and the radiation process of this event is discussed based on these observational features.
\end{abstract}

Keywords. Sun: radio radiation, flares, coronal mass ejections (CMEs), activity

\section{Introduction of the active region}

At $0203-0304$ UT of 2004 November 10 the radio burst occurred in the active region AR10696, which has been one of the comparatively strong energetic active regions since the 23rd solar cycle and during its stay on the solar disc there occurred $45 \mathrm{H} \alpha$ flare, located at (N09, W49), among which there were one 3B flare, 2 flares of Importance 2, 4 flares of Importance 1 and 38 subflares, and in the radio wave band (the meterwave, decimetric wave and microwave wavelengths) there also occurred the large bursts of complex type and CMEs many times.

\section{Observation and analysis}

\subsection{Observed data}

The enhanced radio radiation of the event of 2004 November 10 was observed in the frequency range from $34000 \mathrm{MHz}$ to $18 \mathrm{MHz}$ in the lower coronal. The observed data of the 14 wave bands $(245,410,500,610,986,1161,1336,1415,2695,4995,8800,15400$, 17000 and $34000 \mathrm{MHz}$ ) selected from the frequency range are used to make the analysis with those of the $\mathrm{H} \alpha$ flares and CMEs. This event was the great burst of multispike and complex type during the whole spectrum. The detailed analysis of a great burst of $47 \mathrm{~GB}$ type recorded at the National Astronomical Observatories/Yunnan Observatory in the frequency range of 625 to $1500 \mathrm{MHz}$ is principally made in this present article (see Fig. 1). From Fig. 1 it can be seen that the burst begins in the vicinity of 0202 UT, reaches its maximum at 0206 UT and ends near 0233 UT, with the peak flux being 1776 sfu.

The $\mathrm{H} \alpha$ flares and radio materials used in this paper are take from the data of Solar Geophysical Data (SGD).

\subsection{Tim distribution of the burst}

From the data we analyze it can be seen that the meter-wave burst started at 02060207 UT, the decimetric wave burst began at 0203-0204 UT and the microwave burst commenced at 0203-0205 UT. The H $\alpha$ flare began at 0204 UT, reached its maximum at 
0210UT and ended at 0315 UT, located at (N09, W49). The CME occurred at 0226 UT, Accompanied by the type II burst. Based on the difference of the starting time of the bursts at different frequencies it can be reckoned that the place of the initial electron acceleration and energy release of the event was in the vicinity of the decimetric waveband (986-1336 MHz).

\subsection{Intensity distribution of the burst}

The radiant intensity of the burst at $610 \mathrm{MHz}$ firstly reached a peak value, $6100 \mathrm{sfu}$, and two minutes later, the peak value at $15400 \mathrm{MHz}$ reached the maximum, $6800 \mathrm{sfu}$, which occurred in the impulsive phase of the 3B flare. The CME which occurred at 0226 UT was in the full halo state (the angular breadth was 360 degrees), with the velocity reaching $3387 \mathrm{~km} / \mathrm{s}$. The slow-drift type II burst was also reached at Culgoora, Astralia, in the low frequency range.

\subsection{Fine structures of the decimetric waves}

From Figure 1, we can see that there are several groups of normal and reverse type III bursts with the duration of hundreds of ms and drift rate of hundreds of $\mathrm{MHz} / \mathrm{s}$. This type III bursts have a starting frequency of about $1128 \mathrm{MHz}$ and ending frequency of about $1210 \mathrm{MHz}$. There are no type III burst beyond the frequency range from 1000-1215 MHz.
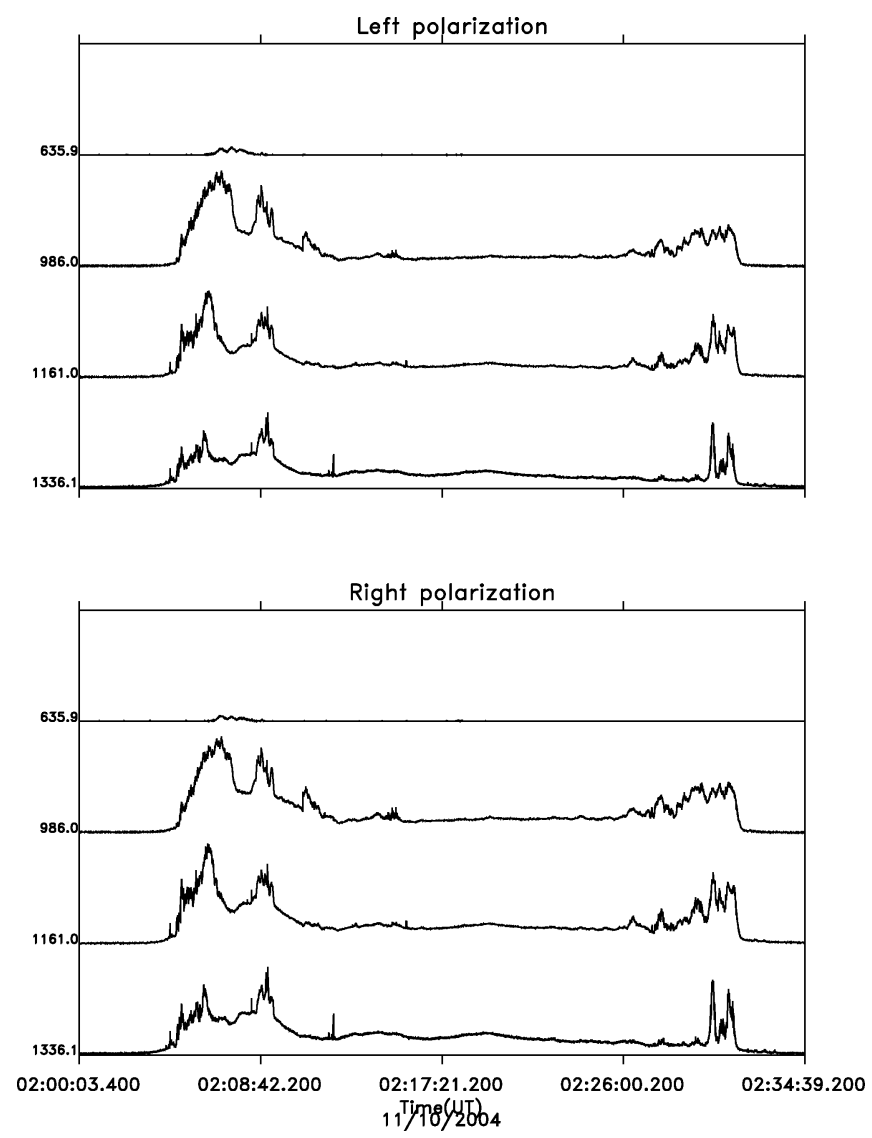

Figure 1. The solar radio burst on 2004 November 10. 


\section{Discussions}

This burst had quite broad radiant frequency range, showing that there should exist a very large radiant region. The broad band or impulsive microwave bursts with long or short duration generally stem from the closed magnetic field in the low corona active regions and are all attributed to the synchro-cyclotron radiation of non-thermal energetic electrons (Vourlidas, 2004, Yan et al., 2007, Huang and Nakajima, 2009), while the bursts at metric and decimetric wavelengths possibly originate from plasma radiation. In addition, in the coronal the propagation of the flare blast wave and the instability of the ensuing interaction of it with the prestored large-scale magnetic structure probably lead to the type II radio bursts observed in the range of metric waves (Wang et al., 2002, Pohjolainen et al., 2001). The meter-wave and decimetric wave type II bursts most probably result from the plasma radiation on the wave front of the MHD shock waves (Pohjolainen et al., 2005).

We hold that the electron acceleration of the flare impulsive phase is caused by the electric field or electrostatic wave in the magnetic reconnection. During the impulsive phase the reconnection may be composed of lots of small current sheets and in the wave bands near the reconnection the peak value of the burst may be the multiple superposition of minimal spikes while at the place far away from the acceleration because of the degradation and diffusion of the accelerated electrons in the propagation these small spikes will be smoothed more and more, showing that the decay phase after the magnetic reconnection is the turbulence acceleration process. Thus, in the burst flux cross-sections of different wavelengths in the impulsive phase, the place where there are much more spikes may be that much closer to the magnetic reconnection electron acceleration in the flare. For example, at $986-1336 \mathrm{MHz}$, in the burst curves of the three wave bands there are the most burst peak values at $1194 \mathrm{MHz}$ which should be much closer to the electron acceleration region.

In a word, the radiation process of a great burst with a long duration which occurs at metric, decimetric and microwave wavelengths is very complicated, not a homogeneous radiation mechanism but the radiation process where there exists the combination simultaneously.

\section{Acknowledgements}

This research is supported by the NSFC grants (Nos. 10473020 and 10333030) and the foundation of the Chinese Academy of Sciences. It is also supported by the Project of Cultivating Western Talents of China.

\section{References}

Huang, G. L. \& Nakajima, H. 2009, ApJ, 702, 19

Kliem, B., Karlicky, M., \& Benz, A. O. 2000, A\& A, 360, 715

Pohjolainen, S., Vilmer, N., \& Khan, J. I. 2005, A\&A, 434, 329

Pohjolainen, S., Maia, D. J. F., Pick, M. et al., 2001, ApJ, 556, 421

Vourlidas, A. 2004, In Solar and Space Weather Radiophysics, 223

Willson, R. F. 2004, Solar Physics, 197, 399

Wang, H. M., Gallagher, P., \& Yurchyshyn, V. 2002, ApJ, 69, 26

Yan, Y. H., Huang, J., Chen, B., \& Sakurai, T. 2007, PASJ, 59, 815 